\title{
Numerical Schemes for Stochastic Differential Equations with Variable and Distributed Delays: The Interpolation Approach
}

\author{
Xueyan Zhao, Feiqi Deng, and Shifang Kuang \\ Systems Engineering Institute, South China University of Technology, Guangzhou 510640, China \\ Correspondence should be addressed to Xueyan Zhao; zhaoxueyan2005@163.com
}

Received 10 October 2013; Accepted 30 December 2013; Published 24 February 2014

Academic Editor: Patricia J. Y. Wong

Copyright (C) 2014 Xueyan Zhao et al. This is an open access article distributed under the Creative Commons Attribution License, which permits unrestricted use, distribution, and reproduction in any medium, provided the original work is properly cited.

\begin{abstract}
A kind of the Euler-Maruyama schemes in discrete forms for stochastic differential equations with variable and distributed delays is proposed. The linear interpolation method is applied to deal with the values of the solutions at the delayed instants. The assumptions of this paper on the coefficients and related parameters are somehow weaker than those imposed by the related past literature. The error estimations for the Euler-Maruyama schemes are given, which are proved to be the same as those for the fundamental EulerMaruyama schemes.
\end{abstract}

\section{Introduction}

It is well known that most stochastic differential equations (SDEs) arising in many applications are nonlinear and cannot be solved explicitly, so the construction of efficient computational methods is of great importance. Hence, in the past decades, the numerical approximation schemes for SDEs have been widely investigated and a lot of fundamental results have been obtained [1-6]. Among the proposed methods, the Euler-Maruyama scheme is one of the most typical schemes. This method has been generalized to some complex SDEs, for example, SDEs with delays terms [7-14], the neutral terms [15], the impulsive terms [16], the terms with Markovian switching $[17,18]$, and the equations driven by Poisson's processes [19-21]. Of course, the approximation schemes for SDEs have been generalized to the SDEs with variable delays and some classical results have been obtained [22-24].

For the case with variable delays, the main difficulty in the construction of the approximation schemes is how to estimate the values of the solutions at the delayed instants. To overcome this problem, $[11,22]$ proposed to use the approximate values at the nearest grid points on the left of the delayed arguments. That amounted to a simple interpolation of the undetermined approximate values of the solutions at nonmesh points by piecewise constant polynomials whose values are taken at the left endpoints of the intervals containing the delayed arguments. Of course, by this method, some variants of the Euler-Maruyama schemes for SDEs have been obtained [12, 13, 18, 19, 21, 24].

In this paper, we consider the time-varying stochastic models with both variable delays and distributed delays. To approximate the values of the solutions at the delayed times, we use an interpolation method. To find the approximate values of the involved integrals, we use the rectangular method or the trapezoidal method. It should be pointed out that our model is a kind of time-varying one, we impose no Hölder continuity for the initial data in this paper, and our schemes are in the discrete forms, which are practical in real applications.

\section{Preliminaries}

In the paper, let $\left(\Omega, \mathscr{F}_{t},\left\{\mathscr{F}_{t}\right\}_{t \geq 0}, \mathscr{P}\right)$ be a complete probability space with a filtration $\left\{\mathscr{F}_{t}\right\}_{t \geq 0}$ satisfying the usual conditions; that is, it is right continuous and $\mathscr{F}_{0}$ contains all $P$-null sets. Let $W(t)=\left[W_{1}(t), W_{2}(t), \ldots, W_{m}(t)\right]^{T}$ be an $m$ dimensional Wiener process defined on the probability space $\left(\Omega, \mathscr{F},\left\{\mathscr{F}_{t}\right\}_{t \geq 0}, \mathscr{P}\right)$. $\tau$ will be a positive constant which stands for the upper bound for the bounded delays involved in the equations, and $I_{\tau}=[-\tau, 0] . C\left(I_{\tau}, R^{n}\right)$ denotes the space of continuous functions $\phi$ from $I_{\tau}$ to $R^{n}$ with norm $|\phi|=$ $\sup _{\theta \in I_{\tau}}\|\phi(\theta)\|$, where $\|\cdot\|$ is any kind of norms for vectors. Let $t_{0} \in R^{+}=[0,+\infty)$ and $T$ be two instants with $0 \leq t_{0}<T, I=$ $\left[t_{0}-\tau, T\right]$. For a given function $x(t) \in C\left(I, R^{n}\right)$, the associated 
function $x_{t} \in C\left(I_{\tau}, R^{n}\right)$ is defined as $x_{t}(\theta)=x(t+\theta), \theta \in I_{\tau}$. Denote $E\left(p, t_{0}, T\right)=E\left\{\sup _{t_{0}-\tau \leq s \leq T}\left\|x\left(s, t_{0}, \phi_{0}\right)\right\|^{p}\right\}$.

For the general theory of functional differential equations, the readers are referred to [25], and for the general theory of stochastic functional differential equations the readers are referred to [6].

Given an Itô SDE with variable and distributed delays

$$
\begin{gathered}
\mathrm{d} x(t)=f\left(t, x(t), x\left(t-\tau_{1}(t)\right), \ldots, x\left(t-\tau_{N}(t)\right),\right. \\
\left.\quad \int_{-\tau_{A}(t)}^{0} A(t, \theta) x(t+\theta) \mathrm{d} \theta\right) \mathrm{d} t \\
+g\left(t, x(t), x\left(t-\tau_{1}(t)\right), \ldots, x\left(t-\tau_{N}(t)\right),\right. \\
\left.\quad \int_{-\tau_{F}(t)}^{0} F(t, \theta) x(t+\theta) \mathrm{d} \theta\right) \mathrm{d} W(t), \\
x_{t_{0}}(\theta)=\phi_{0}(\theta), \quad \theta \in I_{\tau},
\end{gathered}
$$

where $t \in\left[t_{0}, T\right]$ and the state $x \in R^{n}$, the solution $x_{t}$ is a stochastic process in $C\left(I_{\tau}, R^{n}\right)$, with the given initial data $\phi_{0}=$ $\left\{\phi_{0}(\theta): \theta \in I_{\tau}\right\} \in C\left(I_{\tau}, R^{n}\right) . f: R^{+} \times R^{n} \times \cdots \times R^{n} \rightarrow R^{n}$ and $g: R^{+} \times R^{n} \times \cdots \times R^{n} \rightarrow R^{n \times m}$ are continuous in their arguments, $0 \leq \tau_{i}(t), \tau_{A}(t), \tau_{F}(t) \leq \tau, i=1,2, \ldots, N$. We have that $A(t, \theta), F(t, \theta) \in R^{n \times n}$. Define $\tau_{0}(t)=0$.

The numerical scheme of (1) has not been investigated so far in the related literature; we will propose a kind of numerical schemes for (1), with fewer assumptions for its parameters, that is, the coefficients, the delays, and the initial data, except the ordinary basic assumptions.

Assume that the coefficients $f$ and $g$ satisfy the local Lipschitz condition and linear growth condition.

$\left(\mathrm{H}_{1}\right)$ Local Lipschitz condition: for arbitrary given positive number $\alpha$, there is a related constant $L_{\alpha}>0$ such that

$$
\begin{aligned}
& \| f\left(t, \widehat{x}_{0}, \widehat{x}_{1}, \widehat{x}_{2}, \ldots, \widehat{x}_{N}, \widehat{x}_{N+1}\right) \\
& -f\left(s, x_{0}, x_{1}, x_{2}, \ldots, x_{N}, x_{N+1}\right) \|^{2} \\
& \quad \vee \| g\left(t, \widehat{x}_{0}, \widehat{x}_{1}, \widehat{x}_{2}, \ldots, \widehat{x}_{N}, \widehat{x}_{N+1}\right) \\
& \quad-g\left(s, x_{0}, x_{1}, x_{2}, \ldots, x_{N}, x_{N+1}\right) \|^{2} \\
& \leq L_{\alpha}\left(|t-s|^{2}+\sum_{i=0}^{N+1}\left\|\widehat{x}_{i}-x_{i}\right\|^{2}\right),
\end{aligned}
$$

for all $t, s>0$ and $x_{i}, \widehat{x}_{i} \in R^{n}$ with $\left\|x_{i}\right\| \vee\left\|\widehat{x}_{i}\right\| \leq \alpha$, $i=0,1,2, \ldots, N+1$.

$\left(\mathrm{H}_{2}\right)$ Linear growth condition: there exists a positive constant $L$ such that

$$
\begin{aligned}
\| f(t & \left., x_{0}, x_{1}, x_{2}, \ldots, x_{N}, x_{N+1}\right) \|^{2} \\
& \vee\left\|g\left(t, x_{0}, x_{1}, x_{2}, \ldots, x_{N}, x_{N+1}\right)\right\|^{2} \\
& \leq L\left(1+\sum_{i=0}^{N+1}\left\|x_{i}\right\|^{2}\right),
\end{aligned}
$$

for all $t>0$ and $x_{i} \in R^{n}, i=0,1,2, \ldots, N+1$.
The other parameters $\tau(t), A(t, \theta)$, and $F(t, \theta)$ satisfy the following Lipschitz conditions.

$\left(\mathrm{H}_{3}\right)$ For $t, s>0$, there exists a constant $\rho$ such that

$$
\left|\tau_{i}(t)-\tau_{i}(s)\right| \leq \rho|t-s|, \quad i=1,2, \ldots, N .
$$

$\left(\mathrm{H}_{4}\right)$ There exists a constant $\rho^{\prime}$ such that

$$
\left\|A\left(t^{*}, \theta^{*}\right)-A\left(t_{*}, \theta_{*}\right)\right\|^{2} \vee\left\|F\left(t^{*}, \theta^{*}\right)-F\left(t_{*}, \theta_{*}\right)\right\|^{2} \leq \rho^{\prime} \beta^{2},
$$

for $t^{*}, t_{*}>0, \theta^{*}, \theta_{*} \in I_{\tau}$, with $\left|t^{*}-t_{*}\right| \vee\left|\theta^{*}-\theta_{*}\right| \leq \beta$.

Remark 1. In fact, if $f$ and $g$ are such that $f(t, 0,0,0, \ldots, 0,0)$ and $g(t, 0,0,0, \ldots, 0,0)$ are bounded, the local Lipschitz condition $\left(\mathrm{H}_{1}\right)$ implies the linear growth condition $\left(\mathrm{H}_{2}\right)$, where

$$
\begin{aligned}
L=2 \sup _{t \in R^{+}}\{ & \|f(t, 0,0,0, \ldots, 0,0)\|^{2} \\
& \left.\vee\|g(t, 0,0,0, \ldots, 0,0)\|^{2} \vee L_{\alpha}\right\} .
\end{aligned}
$$

It is known that [6], under assumptions $\left(\mathrm{H}_{1}\right)$ and $\left(\mathrm{H}_{2}\right)$, there exists a unique continuous solution on $t \geq t_{0}-\tau$ to (1) for each initial datum $\left(t_{0}, \phi_{0}\right) \in R^{+} \times C\left(I_{\tau}, R\right)$, which is denoted by $x\left(t, t_{0}, \phi_{0}\right)$ in this paper, satisfying

$$
E\left\{\sup _{t_{0}-\tau \leq s \leq t}\left\|x\left(s, t_{0}, \phi_{0}\right)\right\|^{p}\right\}<+\infty \quad \text { on } t \geq t_{0},
$$

for arbitrary constant $p>0$.

\section{The Variant Euler-Maruyama Approximation for SDEs with Variable and Distributed Delays}

Without loss of generality, let $h$ be the step size with $0<h \leq \tau$. Let us take a partition for the existing interval $I=\left[t_{0}-\tau, T\right]$ of the solution as

$$
t_{k}=t_{0}+k h, \quad k=-r_{0}, \ldots,-1,0,1,2, \ldots, K,
$$

where $r_{0}=[\tau / h]$ and $[\cdot]$ is the floor function. In this paper, we take the partition as $T-t_{0}=K h$.

For the interval $\left[t_{k}, t_{k+1}\right]$, by (1) we have

$$
x\left(t_{k+1}\right)=x\left(t_{k}\right)+\int_{t_{k}}^{t_{k+1}} \widehat{f}(s) \mathrm{d} s+\int_{t_{k}}^{t_{k+1}} \widehat{g}(s) \mathrm{d} W(s),
$$

where

$$
\begin{aligned}
& \widehat{f}(s)=f\left(s, x(s), \ldots, \int_{-\tau_{A}(s)}^{0} A(s, \theta) x(s+\theta) \mathrm{d} \theta\right), \\
& \widehat{g}(s)=g\left(s, x(s), \ldots, \int_{-\tau_{A}(s)}^{0} A(s, \theta) x(s+\theta) \mathrm{d} \theta\right) .
\end{aligned}
$$

For variable delay, the points $t_{n}-\tau_{t_{n}}$ may not hit a previous time step. In consequence, there is no previously calculated 
approximate value of the solution available. To overcome this problem, we propose to take the linear interpolation of values of the function involved at the two endpoints of an interval as the approximate value of the function at a point of the interval.

With the above analytic derivation, denote

$$
\begin{gathered}
K_{v_{k, i}}=\frac{1}{h}\left(\left(k-v_{k, i}\right) h-\tau_{i}\left(t_{0}+k h\right)\right), \\
v_{k, i}=\left[\frac{1}{h}\left(k h-\tau_{i}\left(t_{0}+k h\right)\right)\right], \quad i=1,2, \ldots, N, \\
K_{r_{A, k}}^{*}=\frac{\left(r_{A, k}-1\right) h-\tau_{A}\left(t_{0}+k h\right)}{h}, \\
K_{r_{F, k}^{*}}^{*}=\frac{\left(r_{F, k}-1\right) h-\tau_{F}\left(t_{0}+k h\right)}{h}, \\
r_{A, k}=\left[\frac{1}{h}\left(\tau_{A}\left(t_{0}+k h\right)\right)\right], \quad r_{F, k}=\left[\frac{1}{h}\left(\tau_{F}\left(t_{0}+k h\right)\right)\right] .
\end{gathered}
$$
$h$ is

Our variant Euler-Maruyama scheme for (1) with step size

$$
\begin{array}{r}
x_{k+1}=x_{k}+f\left(t_{k}, x_{k}, x_{k, 1}^{*}, x_{k, 2}^{*}, \ldots, x_{k, N}^{*}, X_{k}^{A}\right) h \\
+g\left(t_{k}, x_{k}, x_{k, 1}^{*}, x_{k, 2}^{*}, \ldots, x_{k, N}^{*}, X_{k}^{F}\right) \Delta W_{k}, \\
k=0,1, \ldots, K-1,
\end{array}
$$

where

$$
\begin{gathered}
x_{k, i}^{*}=x_{v_{k, i}}+K_{v_{k, i}}\left(x_{v_{k, i}+1}-x_{v_{k, i}}\right), \\
x_{k-r_{A, k}}^{*}=x_{r_{A, k}-1}+K_{r_{A, k}}^{*}\left(x_{r_{A, k}}-x_{r_{A, k}-1}\right), \\
x_{k-r_{F, k}}^{*}=x_{r_{F, k}-1}+K_{r_{F, k}}^{*}\left(x_{r_{F, k}}-x_{r_{F, k}-1}\right), \\
X_{k}^{A}=h \sum_{j=1}^{r_{A, k}-1} A\left(t_{0}+k h,-j h\right) x_{k-j} \\
+\frac{1}{2}\left(A\left(t_{0}+k h, 0\right) x_{k}+A\left(t_{0}+k h,-r_{A, k} h\right) x_{k-r_{A, k}}\right) h \\
+\frac{1}{2}\left(r_{A, k} h-\tau_{A}\left(t_{0}+k h\right)\right) \\
\times\left(A\left(t_{0}+k h,-r_{A, k} h\right) x_{k-r_{A, k}}\right. \\
\left.+A\left(t_{0}+k h,-\tau_{A}\left(t_{0}+k h\right)\right) x_{k-r_{A, k}}^{*}\right), \\
X_{k}^{F}=h \sum_{j=1}^{r_{F, k}-1} F\left(t_{0}+k h,-j h\right) x_{k-j} \\
+\frac{1}{2}\left(F\left(t_{0}+k h, 0\right) x_{k}+F\left(t_{0}+k h,-r_{F, k} h\right) x_{k-r_{F, k}}\right) h \\
+\frac{1}{2}\left(r_{F, k} h-\tau_{F}\left(t_{0}+k h\right)\right)
\end{gathered}
$$

Remark 4. If we approximate the involved integrals by the rectangular method, then

$$
\begin{aligned}
X_{k}^{A}= & h \sum_{j=1}^{r_{A, k}} A\left(t_{0}+k h,-j h\right) x_{k-j} \\
& +\left(r_{A, k} h-\tau_{A}\left(t_{0}+k h\right)\right) \\
& \times A\left(t_{0}+k h,-\tau_{A}\left(t_{0}+k h\right)\right) x_{k-r_{A, k}}^{*}, \\
X_{k}^{F}= & h \sum_{j=1}^{r_{F, k}} F\left(t_{0}+k h,-j h\right) x_{k-j} \\
& +\left(r_{F, k} h-\tau_{F}\left(t_{0}+k h\right)\right) \\
& \times F\left(t_{0}+k h,-\tau_{F}\left(t_{0}+k h\right)\right) x_{k-r_{F, k}}^{*},
\end{aligned}
$$

where $x_{k-r_{A, k}}^{*}$ and $x_{k-r_{F, k}}^{*}$ are the same as those defined in (13).

\section{Mean Square Estimation for Truncation Errors of the Euler-Maruyama Schemes}

In this section, we analyze the local and global truncation errors of the Euler-Maruyama schemes. For the sake of clarity, we take the case that the integrals $\int_{-\tau_{A}\left(t_{k}\right)}^{0} A\left(t_{k}, \theta\right) x\left(t_{k}+\right.$ $\theta) \mathrm{d} \theta$ and $\int_{-\tau_{F}\left(t_{k}\right)}^{0} F\left(t_{k}, \theta\right) x\left(t_{k}+\theta\right) \mathrm{d} \theta$ are approximated by the 
trapezoidal method. We describe the truncation errors in mean square and analyze the errors directly by the discrete schemes themselves.

4.1. Notations for Error Estimations. The notations proposed here will stand for errors, parameters, and coefficients, respectively, and the coefficients will be used to express the error estimations and the parameters will be used to express the coefficients.

(1) Firstly, as mentioned above, we have an estimation

$$
\begin{aligned}
E\|x(t)\|^{2} \leq E_{T}=: E\left(2, t_{0}, T\right) & =\text { constant, } \\
\text { for } t & \in\left[t_{0}-\tau, T\right] .
\end{aligned}
$$

Define $x_{k, i}^{*}, i=1,2, \ldots, N$, and $X_{k}^{A}, X_{k}^{F}$ for the case $k=$ $-r_{0}, \ldots,-1,0$ similarly to the cases for $k=1,2, \ldots, K$. By the given initial data and the linear interpolation method, we know that $e_{k}^{g}(h)=E\left\|x_{k}-x\left(t_{k}\right)\right\|^{2}=0, k=-r_{0}, \ldots,-1,0$, and $E\left\|x_{k}\right\|^{2} \leq E_{T}, E\left\|x_{k, i}^{*}\right\|^{2} \leq 2 E_{T}$, and $E\left\|X_{k}^{A}\right\|^{2} \vee E\left\|X_{k}^{F}\right\|^{2} \leq$ $3\left(\tau^{2}+2 h^{2}\right) A_{F} E_{T} \leq 9 \tau^{2} A_{F} E_{T}$, for $k=-r_{0}, \ldots,-1,0$.

Define an upper bound sequence $\left\{B_{k}\right\}$ by the iteration

$$
\begin{array}{r}
B_{k+1}=3\left(1+L\left(1+\left(1+2 N+9 \tau^{2} A_{F}\right)\right) h(1+h)\right) B_{k}, \\
B_{0}=E_{T} .
\end{array}
$$

It is obvious that $B_{k}=\lambda^{k}(h) E_{T}$, where $\lambda(h)=3(1+L(1+$ $\left.\left.\left(1+2 N+9 \tau^{2} A_{F}\right)\right) h(1+h)\right)$, and the sequence $\left\{B_{k}\right\}$ is increasing, $E_{T} \leq B_{k} \leq B_{T}, k=0,1, \ldots, K$.

Denote $B_{T}=B_{K}$.

Based on these, we also have the following sets of notations.

(2) Truncation errors of the schemes: consider

$$
\begin{gathered}
e_{k}^{g}(h)=E\left\|x_{k}-x\left(t_{k}\right)\right\|^{2}, \\
\bar{e}_{k}^{g}(h)=\max _{1 \leq q \leq k} E\left\|x_{q}-x\left(t_{q}\right)\right\|^{2}, \\
e_{k, i}^{*}(s)=E\left\|x_{k i}^{*}-x\left(s-\tau_{i}(s)\right)\right\|^{2}, \quad i=1,2, \ldots, N, \\
e_{A, k}(s)=E\left\|X_{k}^{A}-\int_{-\tau_{A}(s)}^{0} A(s, \theta) x(s+\theta) \mathrm{d} \theta\right\|^{2}, \\
e_{F, k}(s)=E\left\|X_{k}^{F}-\int_{-\tau_{F}(s)}^{0} F(s, \theta) x(s+\theta) \mathrm{d} \theta\right\|^{2}, \\
s \in\left[t_{k}, t_{k}+h\right],
\end{gathered}
$$

for $k=1,2, \ldots, K$. Obviously, $e_{k}^{g}(h)$ corresponds to the analytic truncation error $R\left(h ; t, t_{0}, x_{t}\right)$.

By the way, at the end of this paper, we will give an estimation for the local truncation error of the schemes, which will be denoted by $e_{k}^{l}(h)$, for the case that the integrals involving distributed delays are approximately computed by the trapezoidal method.
(3) Parameters: consider

$$
\begin{gathered}
F_{T}=L\left(1+\left(1+2 N+9 \tau^{2} A_{F}\right) B_{T}\right), \\
A_{F}=\sup _{t \in I, \theta \in I_{\tau}}\left\{\|A(t, \theta)\|^{2} \vee\|F(t, \theta)\|^{2}\right\}, \\
M=1+30(1+2 \tau) L_{\alpha}(N+1)+(1+2 \tau) L_{\alpha} \tau^{2} A_{F}^{2}, \\
M^{\prime}=2(1+2 \tau)\left(\tau+10(N+1)(3+\rho)(1+\tau) F_{T}\right. \\
\left.+4 \tau^{3} \rho^{\prime} E_{T}+16(1+2 \tau) \tau^{2} A_{F}^{2} F_{T}\right) L_{\alpha} .
\end{gathered}
$$

4.2. Lemmas. To obtain mean square estimation for global truncation errors and local truncation errors of the EulerMaruyama schemes, we need the following lemma.

Lemma 5. For the schemes (11)-(14), one has the following estimation:

$$
\begin{gathered}
E\left\|x_{k}\right\|^{2} \leq B_{k}, \quad E\left\|x_{k, i}^{*}\right\|^{2} \leq 2 B_{k}, \\
E\left\|X_{k}^{A}\right\|^{2} \vee E\left\|X_{k}^{F}\right\|^{2} \leq 9 \tau^{2} A_{F} B_{k}, \quad k=0,1, \ldots, K .
\end{gathered}
$$

Proof. We prove the conclusion by induction. By the above analysis, the conclusion is true for $k=0$.

Assume that the conclusion is true for some $k>0$.

By the computation scheme and the Markov property of the solutions of the Itô stochastic differential equations, we have

$$
E\left\|x_{k+1}\right\|^{2} \leq 3\left(E\left\|x_{k}\right\|^{2}+E\left\|f_{k}\right\|^{2} h^{2}+E\left\|g_{k}\right\|^{2} h\right) .
$$

By the computation scheme, one can easily show that, if $E\left\|x_{q}\right\|^{2} \leq B_{k}$ for $-r_{0} \leq q \leq k$, then we have $E\left\|x_{k, i}^{*}\right\|^{2} \leq 2 B_{k}$, $E\left\|X_{k}^{A}\right\|^{2} \leq 9 \tau^{2} A_{F} B_{k}$, and $E\left\|X_{k}^{F}\right\|^{2} \leq 9 \tau^{2} A_{F} B_{k}$. Based on these, we have

$$
\begin{aligned}
E\left\|f_{k}\right\|^{2} & \leq L\left(1+E\left\|x_{k}\right\|^{2}+\sum_{i=1}^{N} E\left\|x_{k i}^{*}\right\|^{2}+E\left\|X_{k}^{A}\right\|^{2}\right) \\
& \leq L\left(1+\left(1+2 N+9 \tau^{2} A_{F}\right) B_{k}\right), \\
& E\left\|g_{k}\right\|^{2} \leq L\left(1+\left(1+2 N+9 \tau^{2} A_{F}\right) B_{k}\right),
\end{aligned}
$$

and then

$$
\begin{aligned}
E\left\|x_{k+1}\right\|^{2} & \leq 3\left(1+L\left(1+\left(1+2 N+9 \tau^{2} A_{F}\right)\right) h(1+h)\right) B_{k} \\
& =B_{k+1} .
\end{aligned}
$$

By induction, the conclusion of the theorem is true: that is, we have the estimation $E\left\|x_{k}\right\|^{2} \leq B_{K} \leq B_{T}$ for $k=$ $1,2, \ldots, K$. 
Remark 6. Of course, at the same time, we have the following estimation:

$$
\begin{gathered}
E\left\|x_{k, i}^{*}\right\|^{2} \leq 2 B_{k} \leq 2 B_{T}, \\
E\left\|X_{k}^{A}\right\|^{2} \vee E\left\|X_{k}^{F}\right\|^{2} \leq 9 \tau^{2} A_{F} B_{k} \leq 9 \tau^{2} A_{F} B_{T}, \\
k=0,1, \ldots, K .
\end{gathered}
$$

Remark 7. Вy $\left(\mathrm{H}_{4}\right)$, for $s \in\left[t_{k}, t_{k}+h\right], k=1,2, \ldots, K$, we have

$$
\begin{aligned}
& E\left\{\| f\left(t_{k}, x_{k}, x_{k, 1}^{*}, x_{k, 2}^{*}, \ldots, x_{k, N}^{*}, X_{k}^{A}\right)\right. \\
& \quad-f\left(s, x_{k}, x_{k, 1}^{*}, x_{k, 2}^{*}, \ldots, x_{k, N}^{*}, X_{k}^{A}\right) \|^{2} \\
& \quad \vee \| g\left(t_{k}, x_{k}, x_{k, 1}^{*}, x_{k, 2}^{*}, \ldots, x_{k, N}^{*}, X_{k}^{F}\right) \\
& \left.\quad-g\left(s, x_{k}, x_{k, 1}^{*}, x_{k, 2}^{*}, \ldots, x_{k, N}^{*}, X_{k}^{F}\right) \|^{2}\right\} \\
& \leq L h^{2} .
\end{aligned}
$$

4.3. Mean Square Estimation for Global Truncation Errors. Now we can state mean square estimation for global truncation errors of the Euler-Maruyama schemes and the order of the Euler-Maruyama schemes.

Theorem 8. If the step size $h$ is taken such that $0<h \leq$ $\tau$, then one has global truncation error estimation $e_{k}^{g}(h)=$ $E\left\|x_{k}-x\left(t_{k}\right)\right\|^{2} \leq h\left(M / M^{\prime}\right)\left(E^{M T}-1\right)$ or say $\bar{e}_{k}^{g}(h) \leq h(M /$ $\left.M^{\prime}\right)\left(E^{M T}-1\right), k=1,2, \ldots, K$; that is, the order of the scheme is $1 / 2$.

Proof. The proof of the theorem is a little more difficult than those reported in the related past literature for error analysis, due to the appearance of the argument $t$ in coefficients $f$ and $g$, as well as the error of the schemes for numerical computations of the involved integrals $\int_{-\tau_{A}\left(t_{k}\right)}^{0} A\left(t_{k}, \theta\right) x\left(t_{k}+\theta\right) \mathrm{d} \theta$ and $\int_{-\tau_{F}\left(t_{k}\right)}^{0} F\left(t_{k}, \theta\right) x\left(t_{k}+\theta\right) \mathrm{d} \theta$. We prove the conclusion of the theorem by induction.

Firstly, for $k=-r_{0}, \ldots,-1,0$, we have $e_{k}^{g}(h)=0$; that is, the conclusion of the theorem is true for $k=-r_{0}, \ldots,-1,0$.

Secondly, for the terms $E\left\|x_{v_{k, j}+1}-x\left(t_{v_{k, i}+1}\right)\right\|^{2}, E \| x\left(t_{v_{k, j}}\right)-$ $x_{v_{k, i}}\left\|^{2}, E\right\| x\left(t_{\gamma_{k, i}+1}\right)-x\left(t_{v_{k, i}}\right) \|^{2}$, and $E\left\|x\left(t_{v_{k, i}}\right)-x\left(s-\tau_{i}(s)\right)\right\|^{2}$, we have, respectively,

$$
\begin{gathered}
E\left\|x\left(t_{v_{k, i}}\right)-x_{v_{k, i}}\right\|^{2} \leq e_{k}^{g}(h), \\
E\left\|x_{v_{k, i}+1}-x\left(t_{v_{k, i}+1}\right)\right\|^{2} \leq e_{k}^{g}(h),
\end{gathered}
$$

due to $v_{k, i}+1 \leq k$.

Denote

$$
\begin{aligned}
& f_{k}=f\left(t_{k}, x_{k}, x_{k, 1}^{*}, x_{k, 2}^{*}, \ldots, x_{k, N}^{*}, X_{k}^{A}\right), \\
& g_{k}=g\left(t_{k}, x_{k}, x_{k, 1}^{*}, x_{k, 2}^{*}, \ldots, x_{k, N}^{*}, X_{k}^{F}\right) .
\end{aligned}
$$

By the definition of $e_{k}^{g}(h)$, we have

$$
\begin{aligned}
e_{k+1}^{g}(h)= & E\left\|x_{k+1}-x\left(t_{k+1}\right)\right\|^{2} \\
\leq & E\left\|x_{k}-x\left(t_{k}\right)\right\|^{2}+E\left\|f_{k} h-\int_{t_{k}}^{t_{k+1}} \widehat{f}(s) \mathrm{d} s\right\|^{2} \\
& +E\left\|g_{k} \Delta W_{k}-\int_{t_{k}}^{t_{k+1}} \widehat{g}(s) \mathrm{d} W(s)\right\|^{2} \\
& +2 E\left(x_{k+1}-x\left(t_{k+1}\right)\right)\left(f_{k} h-\int_{t_{k}}^{t_{k+1}} \widehat{f}(s) \mathrm{d} s\right) \\
& +2 E\left(f_{k} h-\int_{t_{k}}^{t_{k+1}} \widehat{f}(s) \mathrm{d} s\right) \\
& \times\left(g_{k} \Delta W_{k}-\int_{t_{k}}^{t_{k+1}} \widehat{g}(s) \mathrm{d} W(s)\right) \\
& +2 E\left(x_{k+1}-x\left(t_{k+1}\right)\right) \\
& \times\left(g_{k} \Delta W_{k}-\int_{t_{k}}^{t_{k+1}} \widehat{g}(s) \mathrm{d} W(s)\right) .
\end{aligned}
$$

By computations, we have

$$
\begin{aligned}
& 2 E\left(x_{k+1}-x\left(t_{k+1}\right)\right)\left(f_{k} h-\int_{t_{k}}^{t_{k+1}} \widehat{f}(s) \mathrm{d} s\right) \\
& \quad=E \int_{t_{k}}^{t_{k+1}} 2\left(x_{k+1}-x\left(t_{k+1}\right)\right)\left(f_{k}-\widehat{f}(s)\right) \mathrm{d} s \\
& \quad \leq E \int_{t_{k}}^{t_{k+1}}\left(\left(x_{k+1}-x\left(t_{k+1}\right)\right)^{2}+\left(f_{k}-\widehat{f}(s)\right)^{2}\right) \mathrm{d} s \\
& \quad=h E\left\|x_{k}-x\left(t_{k}\right)\right\|^{2}+E \int_{t_{k}}^{t_{k+1}}\left\|f_{k}-\widehat{f}(s)\right\|^{2} \mathrm{~d} s, \\
& E\left(x_{k+1}-x\left(t_{k+1}\right)\right)\left(g_{k} \Delta W_{k}-\int_{t_{k}}^{t_{k+1}} \widehat{g}(s) \mathrm{d} W(s)\right) \\
& \quad=E \int_{t_{k}}^{t_{k+1}}\left(x_{k+1}-x\left(t_{k+1}\right)\right)\left(g_{k}-\widehat{g}(s)\right) \mathrm{d} W(s)=0,
\end{aligned}
$$

and similarly

$$
E\left(f_{k} h-\int_{t_{k}}^{t_{k+1}} \widehat{f}(s) \mathrm{d} s\right)\left(g_{k} \Delta W_{k}-\int_{t_{k}}^{t_{k+1}} \widehat{g}(s) \mathrm{d} W(s)\right)=0 .
$$

Inserting (29) and (30) into (28), then we have

$$
\begin{aligned}
e_{k+1}^{g}(h)= & E\left\|x_{k+1}-x\left(t_{k+1}\right)\right\|^{2} \\
\leq & (1+h) E\left\|x_{k}-x\left(t_{k}\right)\right\|^{2}+2 E\left\|f_{k} h-\int_{t_{k}}^{t_{k+1}} \widehat{f}(s) \mathrm{d} s\right\|^{2} \\
& +E\left\|g_{k} \Delta W_{k}-\int_{t_{k}}^{t_{k+1}} \hat{g}(s) \mathrm{d} W(s)\right\|^{2}
\end{aligned}
$$


By the above notations and the given Lipschitz condition, we have

$$
\begin{aligned}
& E\left\|f_{k}-\widehat{f}(s)\right\|^{2} \\
& \leq 2 E\left(\left\|f_{k}-f\left(s, x_{k}, x_{k, 1}^{*}, x_{k, 2}^{*}, \ldots, x_{k, N}^{*}, X_{k}^{A}\right)\right\|^{2}\right. \\
& \left.\quad+\left\|f\left(s, x_{k}, x_{k, 1}^{*}, x_{k, 2}^{*}, \ldots, x_{k, N}^{*}, X_{k}^{A}\right)-\widehat{f}(s)\right\|^{2}\right) \\
& \leq 2 L_{\alpha} h^{2}+2 L_{\alpha} \sum_{j=0}^{N} E\left\|x_{k j}^{*}-x\left(s-\tau_{i}(s)\right)\right\|^{2} \\
& \quad+2 L_{\alpha} E\left\|X_{k}^{A}-\int_{-\tau_{A}(s)}^{0} A(s, \theta) x(s+\theta) \mathrm{d} \theta\right\|^{2} .
\end{aligned}
$$

Let $i=0,1, \ldots, N, s \in\left[t_{k}, t_{k+1}\right]$; we have

$$
\begin{aligned}
x_{k, i}^{*}- & x\left(s-\tau_{i}(s)\right) \\
= & x_{v_{k, i}}+K_{v_{k, i}}\left(x_{v_{k, i}+1}-x_{v_{k, i}}\right)-x\left(s-\tau_{i}(s)\right) \\
= & K_{v_{k, i}}\left(x_{v_{k, i}+1}-x_{v_{k, i}}\right)+\left(x_{v_{k, i}}-x\left(t_{v_{k, i}}\right)\right) \\
& -\left(x\left(s-\tau_{i}(s)\right)-x\left(t_{v_{k, i}}\right)\right) \\
= & K_{v_{k, i}}\left(\left(x_{v_{k, i}+1}-x\left(t_{v_{k, i}+1}\right)\right)\right. \\
& \left.\quad+\left(x\left(t_{v_{k, i}+1}\right)-x\left(t_{v_{k, i}}\right)\right)+\left(x\left(t_{v_{k, i}}\right)-x_{v_{k, i}}\right)\right) \\
+ & \left(x_{v_{k, i}}-x\left(t_{v_{k, i}}\right)\right)+\left(x\left(t_{v_{k, i}}\right)-x\left(s-\tau_{i}(s)\right)\right) \\
\leq & \left(\left(x_{v_{k, i}+1}-x\left(t_{v_{k, i}+1}\right)\right)\right. \\
& \left.+\left(x\left(t_{v_{k, i}+1}\right)-x\left(t_{v_{k, i}}\right)\right)+\left(x\left(t_{v_{k, i}}\right)-x_{v_{k, i}}\right)\right) \\
& +\left(x_{v_{k, i}}-x\left(t_{v_{k, i}}\right)\right)+\left(x\left(t_{v_{k, i}}\right)-x\left(s-\tau_{i}(s)\right)\right)
\end{aligned}
$$

and thus we have

$$
\begin{array}{rl}
E \| x_{k, i}^{*}- & x\left(s-\tau_{i}(s)\right) \|^{2} \\
\leq 5 & E\left\|x_{v_{k, i}+1}-x\left(t_{v_{k, i}+1}\right)\right\|^{2} \\
& +E\left\|x\left(t_{v_{k, i}+1}\right)-x\left(t_{v_{k, i}}\right)\right\|^{2}+E\left\|x\left(t_{v_{k, i}}\right)-x_{v_{k, i}}\right\|^{2} \\
& \left.+E\left\|x_{v_{k, i}}-x\left(t_{v_{k, i}}\right)\right\|^{2}+E\left\|x\left(t_{v_{k, i}}\right)-x\left(s-\tau_{i}(s)\right)\right\|^{2}\right) \\
=5( & E\left\|x_{v_{k, i}+1}-x\left(t_{v_{k, i}+1}\right)\right\|^{2} \\
& +E\left\|x\left(t_{v_{k, i}+1}\right)-x\left(t_{v_{k, i}}\right)\right\|^{2}+2 E\left\|x\left(t_{v_{k, i}}\right)-x_{v_{k, i}}\right\|^{2} \\
& \left.+E\left\|x\left(t_{v_{k, i}}\right)-x\left(s-\tau_{i}(s)\right)\right\|^{2}\right),
\end{array}
$$

$$
\begin{aligned}
& E\left\|x\left(t_{v_{k, i}+1}\right)-x\left(t_{v_{k, i}}\right)\right\|^{2} \\
& =E\left\|\int_{t_{v_{k, i}}}^{t_{v_{k, i}+h}} \widehat{f}(\mu) \mathrm{d} \mu+\int_{t_{v_{k, i}}}^{t_{v_{k, i}+h}} \widehat{g}(\mu) \mathrm{d} W(\mu)\right\|^{2} \\
& \leq 2 E\left(\left\|\int_{t_{v_{k, i}}}^{t_{v_{k, i}+h}} \hat{f}(\mu) \mathrm{d} \mu\right\|^{2}+\left\|\int_{t_{v_{k, i}}}^{t_{v_{k, i}+h}} \hat{g}(\mu) \mathrm{d} W(\mu)\right\|^{2}\right) \\
& \leq 2 E\left(h \int_{t_{v_{k, i}}}^{t_{v_{k, i}+h}}\|\hat{f}(\mu)\|^{2} \mathrm{~d} \mu+\int_{t_{v_{k, i}}}^{t_{v_{k, i}+h}}\|\widehat{g}(\mu)\|^{2} \mathrm{~d} \mu\right) \\
& \leq 2(1+h) h F_{T}, \\
& E\left\|x\left(t_{v_{k, i}}\right)-x\left(s-\tau_{i}(s)\right)\right\|^{2} \\
& =E\left(\int_{t_{v_{k, i}}}^{s-\tau_{i}(s)} \widehat{f}(\mu) \mathrm{d} \mu+\int_{t_{v_{k, i}}}^{s-\tau_{i}(s)} \widehat{g}(\mu) \mathrm{d} W(\mu)\right)^{2} \\
& \leq 2 E\left(\left\|\int_{t_{v_{k, i}}}^{s-\tau_{i}(s)} \widehat{f}(\mu) \mathrm{d} \mu\right\|^{2}+\left\|\int_{t_{v_{k, i}}}^{s-\tau_{i}(s)} \hat{g}(\mu) \mathrm{d} W(\mu)\right\|^{2}\right) \\
& \leq 2 E\left(h \int_{t_{v_{k, i}}}^{s-\tau_{i}(s)}\|\widehat{f}(\mu)\|^{2} \mathrm{~d} \mu+\int_{t_{v_{k, i}}}^{s-\tau_{i}(s)}\|\widehat{g}(\mu)\|^{2} \mathrm{~d} \mu\right) \\
& \leq 2(2+\rho) h(1+h) F_{T},
\end{aligned}
$$

due to $\left|t_{v_{k, i}}-\left(s-\tau_{i}(s)\right)\right| \leq\left|t_{v_{k, i}}-\left(t_{k}-\tau_{i}\left(t_{k}\right)\right)\right|+\mid\left(t_{k}-\tau_{i}\left(t_{k}\right)\right)-$ $\left(s-\tau_{i}(s)\right) \mid \leq h+h+\rho h=(2+\rho) h$; by (34) then it follows that

$$
E\left\|x_{k, i}^{*}-x\left(s-\tau_{i}(s)\right)\right\|^{2} \leq 5\left(3 e_{k}^{g}(h)+2(3+\rho) h(1+h) F_{T}\right) .
$$

At the same time, by the computation method for $X_{k}^{A}$ and $X_{k}^{F}$, that is, the trapezoidal method, we have decomposition

$$
\begin{aligned}
& X_{k}^{A}-\int_{-\tau_{A}(s)}^{0} A(s, \theta) x(s+\theta) \mathrm{d} \theta=\sum_{j=1}^{r_{A, k}+1} Y_{j}^{k}(s), \\
& X_{k}^{F}-\int_{-\tau_{F}(s)}^{0} F(s, \theta) x(s+\theta) \mathrm{d} \theta=\sum_{j=1}^{r_{F, k}+1} Z_{j}^{k}(s),
\end{aligned}
$$

where

$$
\begin{gathered}
Y_{j}^{k}(s)=\frac{1}{2} h\left(A\left(t_{k},-j h\right) x_{k-j}+A\left(t_{k},-(j-1) h\right) x_{k-j+1}\right) \\
-\int_{-j h}^{-(j-1) h} A(s, \theta) x(s+\theta) \mathrm{d} \theta \\
j=1,2, \ldots, r_{A, k},
\end{gathered}
$$




$$
\begin{aligned}
Z_{j}^{k}(s)= & \frac{1}{2} h\left(F\left(t_{k},-j h\right) x_{k-j}+F\left(t_{k},-(j-1) h\right) x_{k-j+1}\right) \\
& -\int_{-j h}^{-(j-1) h} F(s, \theta) x(s+\theta) \mathrm{d} \theta, \\
& j=1,2, \ldots, r_{F, k}, \\
Y_{r_{A, k}+1}^{k}(s)= & \frac{1}{2}\left(r_{A, k} h-\tau_{A}\left(t_{0}+k h\right)\right) \\
\times & \left(A\left(t_{0}+k h,-r_{A, k} h\right) x_{k-r_{A, k}}\right. \\
& \left.+A\left(t_{0}+k h,-\tau_{A}\left(t_{0}+k h\right)\right) x_{k-r_{A, k}}^{*}\right) \\
& -\int_{-\tau_{A}(s)}^{-r_{A, k} h} A(s, \theta) x(s+\theta) \mathrm{d} \theta, \\
Z_{r_{F, k}+1}^{k}(s)= & \frac{1}{2}\left(r_{F, k} h-\tau_{F}\left(t_{0}+k h\right)\right) \\
& \times\left(F\left(t_{0}+k h,-r_{F, k} h\right) x_{k-r_{F, k}}\right. \\
& \left.+F\left(t_{0}+k h,-\tau_{F}\left(t_{0}+k h\right)\right) x_{k-r_{F, k}}^{*}\right) \\
& -\int_{-\tau_{F}(s)}^{-r_{F, k} h} F(s, \theta) x(s+\theta) \mathrm{d} \theta .
\end{aligned}
$$

For $j=1,2, \ldots, r_{A, k}$, we have

$$
\begin{aligned}
Y_{j}^{k}(s)= & \frac{1}{2} h\left(A\left(t_{k},-j h\right) x_{k-j}+A\left(t_{k},-(j-1) h\right) x_{k-j+1}\right) \\
& -\int_{-j h}^{-(j-1) h} A(s, \theta) x(s+\theta) \mathrm{d} \theta \\
= & \frac{1}{2} h\left(A\left(t_{k},-j h\right)\left(x_{k-j}-x\left(t_{k-j}\right)\right)\right. \\
& \left.+A\left(t_{k},-(j-1) h\right)\left(x_{k-j+1}-x\left(t_{k-j+1}\right)\right)\right) \\
& +\frac{1}{2} h\left(A\left(t_{k},-j h\right) x\left(t_{k-j}\right)\right. \\
& \left.+A\left(t_{k},-(j-1) h\right) x\left(t_{k-j+1}\right)\right) \\
& -\int_{-j h}^{-(j-1) h} A(s, \theta) x(s+\theta) \mathrm{d} \theta \\
= & \frac{1}{2} h\left(A\left(t_{k},-j h\right)\left(x_{k-j}-x\left(t_{k-j}\right)\right)\right. \\
& \left.+A\left(t_{k},-(j-1) h\right)\left(x_{k-j+1}-x\left(t_{k-j+1}\right)\right)\right) \\
& +\int_{-j h \quad}^{-(j-1) h}\left(\frac { 1 } { 2 } \left(A\left(t_{k},-j h\right) x\left(t_{k-j}\right)\right.\right. \\
& \left.+A\left(t_{k},-(j-1) h\right) x\left(t_{k-j+1}\right)\right) \\
& -A(s, \theta) x(s+\theta)) \mathrm{d} \theta
\end{aligned}
$$

$$
\begin{gathered}
=\frac{1}{2} h\left(A\left(t_{k},-j h\right)\left(x_{k-j}-x\left(t_{k-j}\right)\right)\right. \\
\left.+A\left(t_{k},-(j-1) h\right)\left(x_{k-j+1}-x\left(t_{k-j+1}\right)\right)\right) \\
+\frac{1}{2} \int_{-j h}^{-(j-1) h}\left(\left(A\left(t_{k},-j h\right) x\left(t_{k-j}\right)\right.\right. \\
\left.+A\left(t_{k},-(j-1) h\right) x\left(t_{k-j+1}\right)\right) \\
\left.-A(s, \theta)\left(x\left(t_{k-j}\right)+x\left(t_{k-j+1}\right)\right)\right) \mathrm{d} \theta \\
+\frac{1}{2} \int_{-j h}^{-(j-1) h}\left(A(s, \theta)\left(x\left(t_{k-j}\right)+x\left(t_{k-j+1}\right)\right)\right. \\
-2 A(s, \theta) x(s+\theta)) \mathrm{d} \theta \\
=\frac{1}{2} h\left(A\left(t_{k},-j h\right)\left(x_{k-j}-x\left(t_{k-j}\right)\right)\right. \\
\left.+A\left(t_{k},-(j-1) h\right)\left(x_{k-j+1}-x\left(t_{k-j+1}\right)\right)\right) \\
+\frac{1}{2} \int_{-j h}^{-(j-1) h}\left(\left(A\left(t_{k},-j h\right)-A(s, \theta)\right) x\left(t_{k-j}\right)\right. \\
+\left(A\left(t_{k},-(j-1) h\right)-A(s, \theta)\right) \\
\left.\times x\left(t_{k-j+1}\right)\right) \mathrm{d} \theta \\
+\frac{1}{2} \int_{-j h}^{-(j-1) h}\left(A(s, \theta)\left(x\left(t_{k-j}\right)-x(s+\theta)\right)\right. \\
\left.+A(s, \theta)\left(x\left(t_{k-j+1}\right)-x(s+\theta)\right)\right) \mathrm{d} \theta .
\end{gathered}
$$

By the given equation, for $s \in\left[t_{k}, t_{k+1}\right]$ and $j=1,2, \ldots$, $r_{A, k}$, we have

$$
\begin{gathered}
x\left(t_{k-j}\right)-x(s+\theta) \\
=\int_{s+\theta}^{t_{k-j}} \widehat{f}(\mu) \mathrm{d} \mu+\int_{s+\theta}^{t_{k-j}} \widehat{g}(\mu) \mathrm{d} W(\mu), \\
E\left\|x\left(t_{k-j}\right)-x(s+\theta)\right\|^{2} \\
\leq 2\left(\left|t_{k-j}-s-\theta\right|\left|\int_{s+\theta}^{t_{k-j}} E\|\widehat{f}(\mu)\|^{2} \mathrm{~d} \mu\right|\right. \\
\left.\quad+\left|\int_{s+\theta}^{t_{k-j}} E\|\hat{g}(\mu)\|^{2} \mathrm{~d} \mu\right|\right),
\end{gathered}
$$

and then we have

$$
\begin{aligned}
E \| x & \left(t_{k-j}\right)-x(s+\theta) \|^{2} \\
& \leq 2\left(((j+1) h+\tau)^{2} F_{T}+((j+1) h+\tau) F_{T}\right) \\
& \leq 4 h(2 h+1) F_{T},
\end{aligned}
$$


due to $\left|t_{k-j}-s-\theta\right| \leq 2 h$. Similarly we have

$$
\begin{aligned}
E\left\|x\left(t_{k-j+1}\right)-x(s+\theta)\right\|^{2} & \leq 2\left((j h+\tau)^{2} F_{T}+(j h+\tau) F_{T}\right) \\
& \leq 4 h(2 h+1) F_{T}
\end{aligned}
$$

Inserting (40) and (41) into (38), we have

$$
\begin{aligned}
& E\left\|Y_{j}^{k}(s)\right\|^{2} \leq \frac{3}{4} E\left(\left(h ^ { 2 } \left(A\left(t_{k},-j h\right)\left(x_{k-j}-x\left(t_{k-j}\right)\right)\right.\right.\right. \\
& +A\left(t_{k},-(j-1) h\right) \\
& \left.\left.\times x_{k-j+1}-x\left(t_{k-j+1}\right)\right)\right)^{2} \\
& +h \int_{-j h}^{-(j-1) h}\left(\left(A\left(t_{k},-j h\right)-A(s, \theta)\right)\right. \\
& \times x\left(t_{k-j}\right) \\
& +\left(A\left(t_{k},-(j-1) h\right)\right. \\
& \left.-A(s, \theta)) x\left(t_{k-j+1}\right)\right)^{2} \mathrm{~d} \theta \\
& +h \int_{-j h}^{-(j-1) h}\left(A(s, \theta)\left(x\left(t_{k-j}\right)-x(s+\theta)\right)\right. \\
& +A(s, \theta)\left(x\left(t_{k-j+1}\right)\right. \\
& \left.-x(s+\theta)))^{2} \mathrm{~d} \theta\right) \\
& \leq \frac{3}{4}\left(4 h^{2} A_{F}^{2} e_{k}^{g}(h)+4 h^{2} \rho^{\prime} h^{2} E_{T}\right. \\
& \left.+16 h^{3}(2 h+1) A_{F}^{2} F_{T}\right) \\
& =\left(A_{F}^{2} e_{k}^{g}(h)+\rho^{\prime} h^{2} E_{T}+4 h(2 h+1) A_{F}^{2} F_{T}\right) h^{2},
\end{aligned}
$$

and similarly we have

$$
E\left\|Z_{j}^{k}(s)\right\|^{2} \leq\left(A_{F}^{2} e_{k}^{g}(h)+\rho^{\prime} h^{2} E_{T}+4 h(2 h+1) A_{F}^{2} F_{T}\right) h^{2} .
$$

For the same reasons, we have

$$
\begin{aligned}
& E\left\|Y_{r_{A, k}+1}^{k}(s)\right\|^{2} \vee E\left\|Z_{r_{F, k}+1}^{k}(s)\right\|^{2} \\
& \quad \leq\left(A_{F}^{2} e_{k}^{g}(h)+\rho^{\prime} h^{2} E_{T}+4 h(2 h+1) A_{F}^{2} F_{T}\right) h^{2} .
\end{aligned}
$$

Consequently we have

$$
\begin{aligned}
e_{A, k}(s)= & E\left\|X_{k}^{A}-\int_{-\tau_{A}(s)}^{0} A(s, \theta) x(s+\theta) \mathrm{d} \theta\right\|^{2} \\
\leq & \left(r_{A, k}+1\right) \sum_{j=1}^{r_{A, k}+1} E\left\|Y_{j}^{k}(s)\right\|^{2} \\
\leq & \left(r_{A, k}+1\right)^{2} \\
& \times\left(A_{F}^{2} e_{k}^{g}(h)+\rho^{\prime} h^{2} E_{T}+4 h(2 h+1) A_{F}^{2} F_{T}\right) h^{2}, \\
e_{F, k}(s)= & E\left\|X_{k}^{F}-\int_{-\tau_{F}(s)}^{0} F(s, \theta) x(s+\theta) \mathrm{d} \theta\right\|^{2} \\
\leq & \left(r_{F, k}+1\right) \sum_{j=1}^{r_{F, k}+1} E\left\|Z_{j}^{k}(s)\right\|^{2} \\
\leq & \left(r_{F, k}+1\right)^{2} \\
& \times\left(A_{F}^{2} e_{k}^{g}(h)+\rho^{\prime} h^{2} E_{T}+4 h(2 h+1) A_{F}^{2} F_{T}\right) h^{2} .
\end{aligned}
$$

In fact, since $\left(r_{A, k}+1\right) h \vee\left(r_{F, k}+1\right) h \leq \tau+h \leq 2 \tau$, as mentioned above, we have

$$
\begin{aligned}
& e_{A, k}(s) \vee e_{F, k}(s) \\
& \leq 4 \tau^{2}\left(A_{F}^{2} e_{k}^{g}(h)+\rho^{\prime} h^{2} E_{T}+4 h(2 h+1) A_{F}^{2} F_{T}\right) .
\end{aligned}
$$

With the above estimations, for every $k$, we have a common estimation

$$
E\left(f_{k}-\widehat{f}(s)\right)^{2} \leq \gamma_{T}(h)
$$

Similarly we have

$$
E\left(g_{k}-\widehat{g}(s)\right)^{2} \leq \gamma_{T}(h),
$$

and then we obtain

$$
\begin{gathered}
E\left\|f_{k} h-\int_{t_{k}}^{t_{k+1}} \widehat{f}(s) \mathrm{d} s\right\|^{2} \leq h^{2} \gamma_{T}(h), \\
E\left\|g_{k} \Delta W_{k}-\int_{t_{k}}^{t_{k+1}} \hat{g}(s) \mathrm{d} W(s)\right\|^{2} \leq h \gamma_{T}(h),
\end{gathered}
$$

where

$$
\begin{aligned}
\gamma_{T}(h)= & 2 L_{\alpha} h^{2}+10 L_{\alpha}(N+1) \\
& \times\left(3 e_{k}^{g}(h)+2(3+\rho) h(1+h) F_{T}\right) \\
& +8 L_{\alpha} \tau^{2}\left(A_{F}^{2} e_{k}^{g}(h)+\rho^{\prime} h^{2} E_{T}+4 h(2 h+1) A_{F}^{2} F_{T}\right) .
\end{aligned}
$$



have

By iterating and observing that $h(k+1)=t_{k+1} \leq T$, we $e_{k+1}^{g}(h) \leq(1+h) E\left\|x_{k}-x\left(t_{k}\right)\right\|^{2}+h(1+2 h) \gamma_{T}(h)$

$$
\begin{aligned}
\leq & (1+h) e_{k}^{g}(h)+2 h(1+2 h) L_{\alpha} h^{2} \\
& +10 h(1+2 h) L_{\alpha} \\
& \times(N+1)\left(3 e_{k}^{g}(h)+2(3+\rho) h(1+h) F_{T}\right) \\
& +8 h(1+2 h) L_{\alpha} \tau^{2} \\
& \times\left(A_{F}^{2} e_{k}^{g}(h)+\rho^{\prime} h^{2} E_{T}+4 h(2 h+1) A_{F}^{2} F_{T}\right) \\
\leq & \left(1+h+30 h(1+2 h) L_{\alpha}(N+1)\right. \\
& \left.+8 h(1+2 h) L_{\alpha} \tau^{2} A_{F}^{2}\right) e_{k}^{g}(h) \\
& +2 h(1+2 h) L_{\alpha} h^{2}+20 h^{2} \\
& \times(1+2 h) L_{\alpha}(N+1)(3+\rho)(1+h) F_{T} \\
& +8 h(1+2 h) L_{\alpha} \tau^{2}\left(\rho^{\prime} h^{2} E_{T}+4 h(2 h+1) A_{F}^{2} F_{T}\right) \\
= & \left(1+h\left(1+30(1+2 h) L_{\alpha}(N+1)\right.\right. \\
& \left.\left.\quad+(1+2 h) L_{\alpha} \tau^{2} A_{F}^{2}\right)\right) e_{k}^{g}(h) \\
& +2 h(1+2 h) L_{\alpha} h^{2}+20 h^{2}(1+2 h) \\
& \times L_{\alpha}(N+1)(3+\rho)(1+h) F_{T} \\
& +8 h(1+2 h) L_{\alpha} \tau^{2}\left(\rho^{\prime} h^{2} E_{T}+4 h(2 h+1) A_{F}^{2} F_{T}\right)
\end{aligned}
$$$$
\leq\left(1+h\left(1+30(1+2 \tau) L_{\alpha}(N+1)\right.\right.
$$$$
\left.\left.+(1+2 \tau) L_{\alpha} \tau^{2} A_{F}^{2}\right)\right) e_{k}^{g}(h)
$$$$
+2 h(1+2 h) L_{\alpha} h^{2}+20 h^{2}
$$$$
\times(1+2 h) L_{\alpha}(N+1)(3+\rho)(1+h) F_{T}
$$$$
+8 h(1+2 h) L_{\alpha} \tau^{2}\left(\rho^{\prime} h^{2} E_{T}+4 h(2 h+1) A_{F}^{2} F_{T}\right)
$$$$
\leq\left(1+h\left(1+30(1+2 \tau) L_{\alpha}(N+1)\right.\right.
$$$$
\left.\left.+(1+2 \tau) L_{\alpha} \tau^{2} A_{F}^{2}\right)\right) e_{k}^{g}(h)
$$

$$
+2 h^{2} L_{\alpha}(1+2 \tau)
$$$$
\times\left(\tau+10(N+1)(3+\rho)(1+\tau) F_{T}\right.
$$$$
\left.+4 \tau^{3} \rho^{\prime} E_{T}+16(1+2 \tau) \tau^{2} A_{F}^{2} F_{T}\right)
$$$$
=(1+h M) e_{k}^{g}(h)+h^{2} M^{\prime}
$$

$$
\begin{aligned}
& =(1+h M)^{k+1} e_{0}^{g}(h)+h^{2} M^{\prime} \sum_{i=0}^{k}(1+h M)^{i} \\
& =h^{2} M^{\prime} \sum_{i=0}^{k}(1+h M)^{i} \\
& =h^{2} M^{\prime} \frac{(1+h M)^{k+1}-1}{h M} \\
& \leq h \frac{M^{\prime}}{M}\left(E^{M T}-1\right) .
\end{aligned}
$$

The proof is complete.

Remark 9. Besides, by the above derivation, we also have estimations for $E\left\|x_{k}\right\|^{2}$ and $E\left\|x_{k i}^{*}\right\|^{2}$ in another form:

$$
\begin{aligned}
& E\left\|x_{k}\right\|^{2} \leq 2\left(E\left\|x\left(t_{k}\right)\right\|^{2}+E\left\|x_{k}-x\left(t_{k}\right)\right\|^{2}\right) \\
& \leq h \frac{M}{M^{\prime}}\left(E^{M T}-1\right)+E_{T} \leq \tau \frac{M}{M^{\prime}}\left(E^{M T}-1\right)+E_{T}, \\
& E\left\|x_{k i}^{*}\right\|^{2} \leq 2\left(E\left\|x\left(t_{k}-\tau_{i}\left(t_{k}\right)\right)\right\|^{2}+E\left\|x_{k i}^{*}-x\left(t_{k}-\tau_{i}\left(t_{k}\right)\right)\right\|^{2}\right) \\
& \leq 2\left(5\left(3 h \frac{M}{M^{\prime}}\left(E^{M T}-1\right)+2(3+\rho) h(1+h) F_{T}\right)\right. \\
&\left.+E_{T}\right) \\
& \leq 2\left(5\left(3 \tau \frac{M}{M^{\prime}}\left(E^{M T}-1\right)+2(3+\rho) \tau(1+\tau) F_{T}\right)\right. \\
&\left.+E_{T}\right) .
\end{aligned}
$$

4.4. Mean Square Estimation for Local Truncation Errors. With the above derivation, by the way, we can get the local truncation error for the Euler-Maruyama schemes. In fact, replacing the term $C_{k}^{g}(h)$ by 0 in the derivation, we just get an estimation for the local truncation error:

$$
\begin{aligned}
e_{k+1}^{g}(h) \leq & (1+h) E\left\|x_{k}-x\left(t_{k}\right)\right\|^{2}+h(1+2 h) \gamma_{T}(h) \\
\leq & 2 h(1+2 h) L_{\alpha} h^{2}+10 h(1+2 h) \\
& \times L_{\alpha}(N+1)\left(2(3+\rho) h(1+h) F_{T}\right) \\
& +8 h(1+2 h) L_{\alpha} \tau^{2}\left(\rho^{\prime} h^{2} E_{T}+4 h(2 h+1) A_{F}^{2} F_{T}\right) \\
= & C_{l}(h) h^{2},
\end{aligned}
$$

where

$$
\begin{aligned}
C_{l}(h)=2(1+2 h)( & h+10(N+1)(3+\rho)(1+h) F_{T} \\
& \left.+4 \tau^{2}\left(\rho^{\prime} h E_{T}+4(2 h+1) A_{F}^{2} F_{T}\right)\right) L_{\alpha} .
\end{aligned}
$$




\section{Conclusion}

As is pointed out above, one of the most important purposes for numerical computation of stochastic systems is obviously to simulate the dynamic behavior of the solutions of the systems. To this end, we have proposed a kind of the EulerMaruyama schemes, coming from the rectangular method for numerical integrals.

In this paper, we have approximated the integrals involving distributed delays by either the rectangular method or the trapezoidal method and established corresponding computation schemes. We have weakened some of the conditions applied in the related past literature; that is, in this paper, we have desired neither the Lipschitz assumption for the variable delays nor the Hölder continuity for the initial data. Under our weaker assumptions, the order for the global truncation error of the Euler-Maruyama schemes is proved to be $1 / 2$ in the meaning of strong convergence.

Of course, for further investigation, the integrals involving distributed delays can be generalized to the form $\int_{-\tau}^{0}\left[\mathrm{~d}_{\theta} \eta(t, \theta)\right] x_{t}(\theta)$ and we can derive similar computation schemes and obtain the same order global truncation errors for the schemes.

\section{Conflict of Interests}

The authors declare that there is no conflict of interests regarding the publication of this paper.

\section{Acknowledgments}

The authors would like to thank the editor and reviewers for their insightful comments and help as well as the National Natural Science Foundation of China under Grant 61273126, the Natural Science Foundation of Guangdong Province under Grants 10251064101000008 and S2012010009675, and the Research Fund for the Doctoral Program of Higher Education of China under Grant 20130172110027 for their financial support.

\section{References}

[1] P. E. Kloeden and E. Platen, Numerical Solution of Stochastic Differential Equations, vol. 23 of Applications of Mathematics (New York), Springer, Berlin, Germany, 1992.

[2] Y. Komori, Y. Saito, and T. Mitsui, "Some issues in discrete approximate solution for stochastic differential equations," Computers \& Mathematics with Applications, vol. 28, no. 1012 , pp. 269-278, 1994.

[3] E. Platen, "An introduction to numerical methods for stochastic differential equations," Acta Numerica, vol. 8, pp. 195-244, 1999.

[4] G. N. Milstein, Numerical Integration of Stochastic Differential Equations, vol. 313 of Mathematics and Its Applications, Kluwer Academic, Dordrecht, The Netherlands, 1995.

[5] Y. Saito and T. Mitsui, "Stability analysis of numerical schemes for stochastic differential equations," SIAM Journal on Numerical Analysis, vol. 33, no. 6, pp. 2254-2267, 1996.
[6] X. Mao, Stochastic Differential Equations and Their Applications, Horwood Publishing Series in Mathematics \& Applications, Horwood Publishing, Chichester, UK, 1997.

[7] C. T. H. Baker and E. Buckwar, "Numerical analysis of explicit one-step methods for stochastic delay differential equations," LMS Journal of Computation and Mathematics, vol. 3, pp. 315335, 2000.

[8] E. Buckwar, "Introduction to the numerical analysis of stochastic delay differential equations," Journal of Computational and Applied Mathematics, vol. 125, no. 1-2, pp. 297-307, 2000.

[9] U. Küchler and E. Platen, "Strong discrete time approximation of stochastic differential equations with time delay," Mathematics and Computers in Simulation, vol. 54, no. 1-3, pp. 189-205, 2000.

[10] Y. Hu, S. E. A. Mohammed, and F. Yan, Numerical Solution of Stochastic Differential Systems with Memory, Southern Illinois University, 2001.

[11] X. Mao and S. Sabanis, "Numerical solutions of stochastic differential delay equations under local Lipschitz condition," Journal of Computational and Applied Mathematics, vol. 151, no. 1, pp. 215-227, 2003.

[12] X. Mao, "Exponential stability of equidistant Euler-Maruyama approximations of stochastic differential delay equations," Journal of Computational and Applied Mathematics, vol. 200, no. 1, pp. 297-316, 2007.

[13] H. Zhang, S. Gan, and L. Hu, "The split-step backward Euler method for linear stochastic delay differential equations," Journal of Computational and Applied Mathematics, vol. 225, no. 2, pp. 558-568, 2009.

[14] X. Mao, "Numerical solutions of stochastic differential delay equations under the generalized Khasminskii-type conditions," Applied Mathematics and Computation, vol. 217, no. 12, pp. 55125524, 2011.

[15] F. Jiang, Y. Shen, and F. Wu, "A note on order of convergence of numerical method for neutral stochastic functional differential equations," Communications in Nonlinear Science and Numerical Simulation, vol. 17, no. 3, pp. 1194-1200, 2012.

[16] G. Zhao, M. H. Song, and M. Z. Liu, "Exponential stability of Euler-Maruyama solutions for impulsive stochastic differential equations with delay," Applied Mathematics and Computation, vol. 215 , no. 9, pp. 3425-3432, 2010.

[17] S. Zhou and F. Wu, "Convergence of numerical solutions to neutral stochastic delay differential equations with Markovian switching," Journal of Computational and Applied Mathematics, vol. 229, no. 1, pp. 85-96, 2009.

[18] F. Jiang, Y. Shen, and J. Hu, "Stability of the split-step backward Euler scheme for stochastic delay integro-differential equations with Markovian switching," Communications in Nonlinear Science and Numerical Simulation, vol. 16, no. 2, pp. 814-821, 2011.

[19] M. Wei, "Convergence of numerical solutions for variable delay differential equations driven by Poisson random jump measure," Applied Mathematics and Computation, vol. 212, no. 2, pp. 409-417, 2009.

[20] L.-s. Wang and H. Xue, "Convergence of numerical solutions to stochastic differential delay equations with Poisson jump and Markovian switching," Applied Mathematics and Computation, vol. 188, no. 2, pp. 1161-1172, 2007.

[21] L.-s. Wang, C. Mei, and H. Xue, "The semi-implicit Euler method for stochastic differential delay equations with jumps," Applied Mathematics and Computation, vol. 192, no. 2, pp. 567578, 2007. 
[22] X. Mao, "Approximate solutions for a class of stochastic evolution equations with variable delays. II," Numerical Functional Analysis and Optimization, vol. 15, no. 1-2, pp. 65-76, 1994.

[23] M. Milošević, "Highly nonlinear neutral stochastic differential equations with time-dependent delay and the Euler-Maruyama method," Mathematical and Computer Modelling, vol. 54, no. 910, pp. 2235-2251, 2011.

[24] M. Wei, "Convergence analysis of semi-implicit Euler methods for solving stochastic equations with variable delays and random jump magnitudes," Journal of Computational and Applied Mathematics, vol. 235, no. 8, pp. 2569-2580, 2011.

[25] J. K. Hale and S. M. Verduyn Lunel, Introduction to FunctionalDifferential Equations, vol. 99 of Applied Mathematical Sciences, Springer, New York, NY, USA, 1993. 


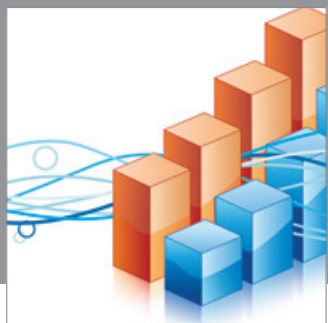

Advances in

Operations Research

mansans

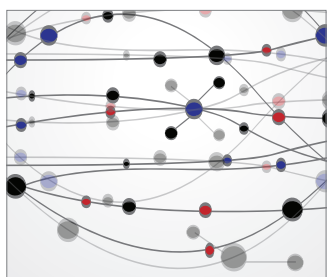

The Scientific World Journal
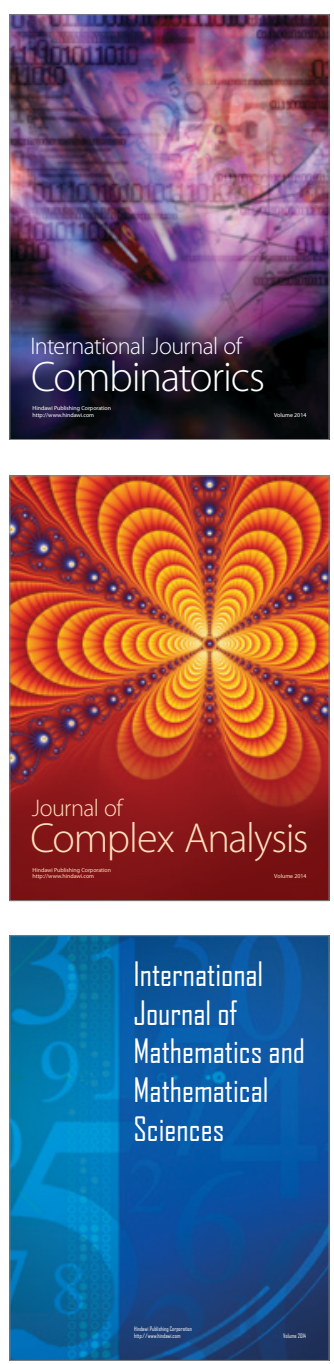
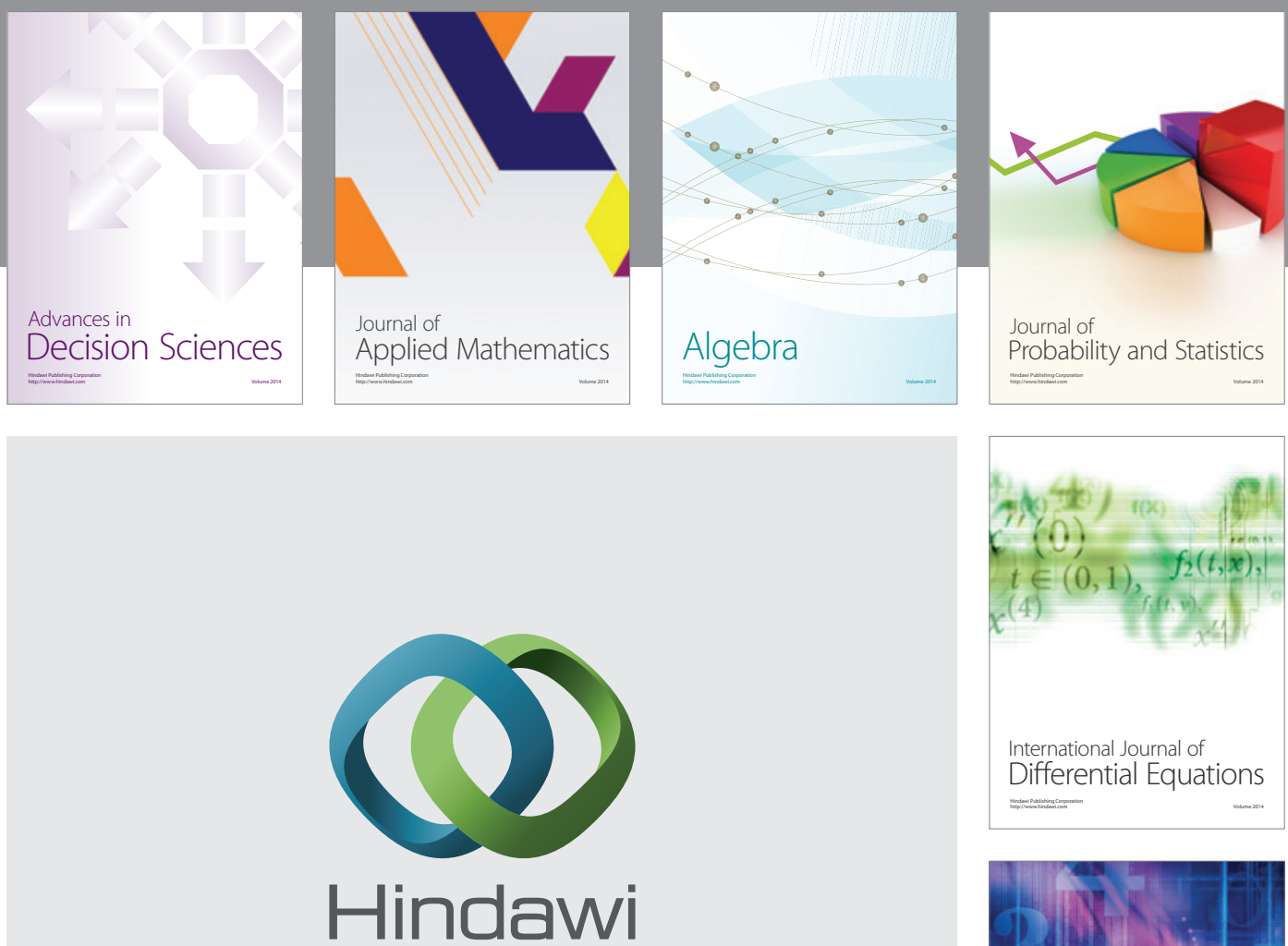

Submit your manuscripts at http://www.hindawi.com
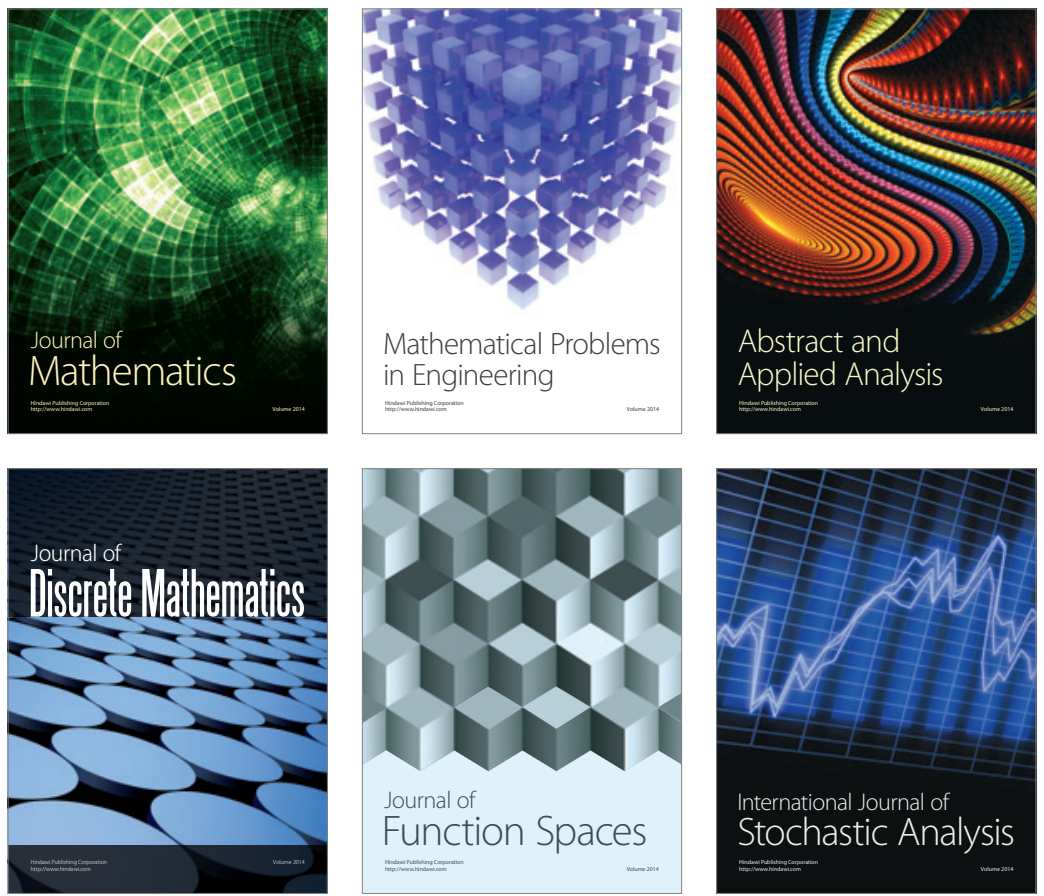

Journal of

Function Spaces

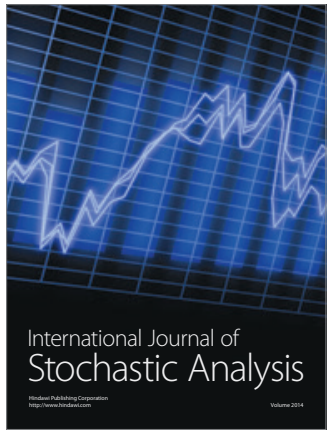

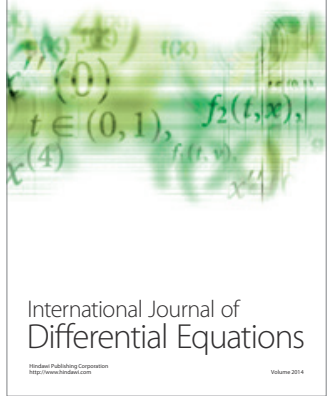
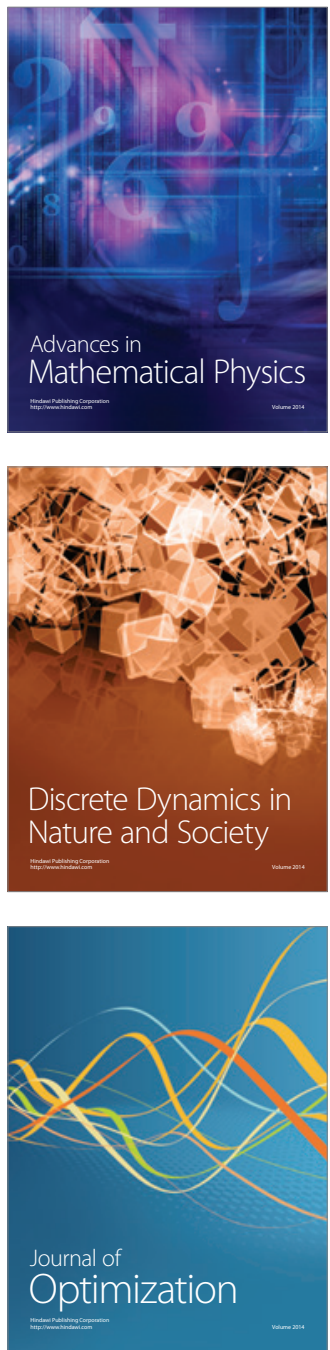